\title{
Short Fatigue Crack Growth at Different Maintenance Times for LZ50 Steel
}

\author{
B. Yang, ${ }^{1}$ B. Q. Ma, Y. X. Zhao, and S. N. Xiao
}

State Key Laboratory of Traction Power, Southwest Jiaotong University, Chengdu, China

1.yb@swjtu.cn

Based on the average fatigue life of LZ50 axle steel specimens without surface rolling, five maintenance times were determined. Accordingly, five groups of specimens were turned and rolled at above maintenance times and were fatigued using a replica technique. The results show that the crack growth rate is much lower than that before rolling at a given dominant short crack size. The effective short crack density of all specimens decreases significantly after maintenance. However, with the postponement of surface maintenance, the highest effective short crack density for the five studied groups of specimens increases continuously, while the average fatigue life decreases gradually. A maintenance time effect function is presented to refine a short crack growth model described previously. The revifined model can include a significant effect of the maintenance time on short crack growth and predict its patterns at different maintenance times with exiting test results for LZ50 axle steel.

Keywords: fatigue, short crack, axle, maintenance, growth rate model.

Introduction. With the increase in speed, transport capacity and traffic density of railway vehicles, their key components have to withstand more heavy-duty service conditions [1]. An important component of vehicle running gear is axle, which bears complicated alternate loads during its operation, has the highest loading frequency, and exhibits the most complex failure modes [2]. Any axle failures caused by fatigue damage may result in train derailing, and the safety of railway operation will be gravely affected. For components with a smooth surface subjected to alternate loading, the initiation, coalescence and propagation of short fatigue cracks (SFC) normally occupy over $70 \%$ of the total fatigue life [3]. For example, with a overhaul cycle of $100,000 \mathrm{~km}$ and a reliability of 0.999 , the critical size of a semi-elliptical crack in the load-relieving groove of $\mathrm{RD}_{2}$ axle used in railway freight cars in China, is $1.23 \mathrm{~mm}$, while the respective size of a circumferential crack is only $0.94 \mathrm{~mm}[4,5]$. Therefore, it can be concluded that the fatigue damage process in such axle occurs mainly at the SFC stage. To describe the SFC growth behavior, numerous studies have been conducted and, accordingly, many SFC growth rate models have been presented, such as the cyclic stress/strain-related model $[6,7]$, the shearing strain model [8], the dislocation based model [9], the general Paris law model [10], and the multi-microstructural barriers' model [11].

The final processing method for the load-relieving groove of RD2 axle has once been turning, the maintenance strategy for it being finish turning and surface rolling. However, the impact of surface treatment time choice on the maintenance effect, i.e., the maintenance time effect on the short fatigue crack growth behavior, is still an open issue.

LZ50 axle steel is widely applied in Chinese railway manufacturing industry, while its production technology complies with the adopted the AAR M-101axle standard [12]. This study is based on a series of fatigue tests of this material by the replica technique. Different maintenance times are chosen for five groups of specimens. The influence of maintenance time on SFC propagation, crack density, and fatigue life is investigated. The deceleration behavior of crack growth at the microstructural crack (MSC) stage is observed in specimens both before and after their surface treating. A revised multi-microstructural barriers' model is proposed to account for the maintenance time effect. 


\section{Experimental Details.}

1.1. Materials and Specimens. The material under study is LZ50 axle steel. Its chemical composition is: $0.47 \mathrm{C}, 0.26 \mathrm{Si}, 0.78 \mathrm{Mn}, 0.021 \mathrm{Al}, 0.02 \mathrm{Cr}, 0.028 \mathrm{Ni}, 0.15 \mathrm{Cu}$, $\leq 0.014 \mathrm{P}, \leq 0.01 \mathrm{~S}$, and the remainder Fe by wt.\%. The static mechanical properties at room temperature are: $209750 \mathrm{MPa}$ for the Young modulus, $656.43 \mathrm{MPa}$ for the tensile strength, $383.57 \mathrm{MPa}$ for the yield strength, $54.71 \%$ for elongation, and $26.57 \%$ for the cross-sectional area reduction. The heat treatment is double normalizing and subsequent tempering, in accordance with the TB 2945-1999 railway standard of China. After heat treatment, the microstructure of material consists of coarse ferrite particles and layered pearlite particles. There is a banded structure, whereas the mean value of intervals between two rich pearlite bands, $d_{2}$, is about $109 \mu \mathrm{m}$ with a high dispersion. Gathering effects exist in both ferrite and pearlite structures. The average equivalent diameter for ferrite grains, $d_{1}$, is $14.6 \mu \mathrm{m}$ [11]. The static microhardness for pearlite of LZ50 axle steel is $223.4 \mathrm{HV}_{0.1}$ while that for ferrite is $191.4 \mathrm{HV}_{0.1}$, which is quite expected, since microhardness of pearlite usually exceeds that of ferrite. In total, 33 smooth axial hourglass shaped specimens with $10 \mathrm{~mm}$ diameter were machined [11].

1.2. Maintenance Simulation. The machining technology for the load-relieving groove of $\mathrm{RD}_{2}$ axle in practice and the maintenance simulation parameters of present research are described in detail in the earlier study [13]. The purpose of finish turning is to remove possible surface cracks, while rolling is meant to strengthen the surface of the load-relieving groove or the specimen. It can be seen that the simulation technology meets the maintenance requirements of a real $\mathrm{RD}_{2}$ axle. After surface rolling, the Vickers hardness of specimens increases from 201.68 to $222.90 \mathrm{HV}_{0.1}$. Meanwhile, the absolute values of axial and circumferential compressive stresses for specimens after surface rolling are higher by 170 and $101 \mathrm{MPa}$, respectively, than those before rolling [13].

1.3. Replication Tests. In short crack research, the replication technique is a widely applied fatigue test method [14]. By utilizing this technique, present tests were performed under a stress-controlled sine wave mode on a Rumul $250 \mathrm{kN}$ high-frequency fatigue test machine at room temperature in air environment. The symmetrical cyclic stress amplitude was $230 \mathrm{MPa}$. To study the relationship between crack propagation and microstructure, specimen surfaces were etched by $4 \%$ nitric acid alcohol, so that their metallographic structure was exposed.

Firstly, all specimens without maintenance were tested according to the replication technique. Secondly, when the number of loading cycles attained the preset maintenance time, the test was interrupted and specimens were dismounted. Thirdly, the above specimens were turned and surface-rolled in compliance with the simulation technology. Finally, specimens after maintenance were mounted on the test machine again and fatigued at $230 \mathrm{MPa}$ by the replication method to their final failure.

Preliminary test results have indicated that the average fatigue life of specimens without maintenance is 137,705 cycles [11]. To investigate the maintenance time effect, surface treatment times for five groups of specimens were determined according to this life estimation. That is, turning and surface rolling were applied when life fraction, $f$, was 0.0 , $0.3,0.5,0.6$, and 0.7 , respectively. Specimens were indexed according to their maintenance time, i.e., S0.0, S0.3, S0.5, S0.6, and S0.7.

Noteworthy is that the axle diameter will be slightly reduced after maintenance, which will lead to the higher local stress level even under the same service loading conditions. Since this study is focused on the influence of surface treatment time and maintenance technology on short fatigue crack behavior, the increase in stress amplitude caused by size reduction at a constant test load can make this analysis more complex. Therefore, in this study, the test load was recalculated based on the specimen actual diameter after maintenance to ensure that the replication test is conducted at the stress level of $230 \mathrm{MPa}$. 
2. Description of Short Crack Behavior. Previous work performed by the authors has indicated that the surface rolling process and the choice of maintenance time would strongly influence the short crack behavior in the aspects of crack growth rate, short crack density, and fatigue life [15]. To facilitate the understanding of these impacts, a brief description is given as follows.

2.1. Dominant Short Crack Growth Rate. Using the approach of the effective short fatigue crack criterion $[16,17]$, the relations between dominant short crack (DSC) growth rate, $d a / d N$, and crack size, $a$, for five different surface rolling times can be derived:

(1) For five groups of specimens, the growth rate sharply decelerates once or twice during the crack growth process. The ferrite grain boundary and the pearlite banded structure restrain the growth of short cracks. These inherent resistances of material do not change with maintenance time.

(2) DSC of unrolled specimens grows much faster than after rolling at the same DSC size, which can be attributed to different surface conditions. Rolling increases both the surface hardness and the absolute value of residual compressive stresses. Thus, fatigue characteristics of unrolled material are not improved, and the respective constraint force for crack initiation and growth is relatively low.

2.2. Effective Short Crack Density. Effective short crack density is defined as the average number of short cracks per unit area in the initial zone of DSC at MSC stage [11]. At the physically short crack (PSC) stage, the observation regions transfer to the two zones ahead of DSC tips. A higher density indicates the formation of microstructural conditions for short crack growth, and also reflects the strengthening of the SFC collective effect, as well as the inherent difference of the local microstructure [18].

A detailed introduction on the changes in effective short crack density with respective fatigue life fraction for S0.0 to S0.7 specimens can be found elsewhere [15]. It is clear that crack density of specimens after maintenance is much less than that of specimens with no surface treatment. Turning and rolling treatments effectively inhibit the collective initiation of short cracks. Crack density of all specimens under study shows the same overall trend, i.e., it increases at MSC stage, decreases at PSC stage, and attains the peak value at the transition point between these two stages. The DSC size according to the density peak value is about the mean value of intervals for rich pearlite bands. In order to empasize the difference of the density before and after maintenance more, Fig. 1 gives two typical replication photos of specimens in both kinds of surface processing states at the transition points between MSC stage and PSC stage. The number of microcracks in specimens before maintenance (Fig. 1a) is much larger than that in specimens after repair (Fig. 1b) at the same stage of the fatigue process [18]. It can be concluded from the above discussion that the rich pearlite banded structure is the strongest microstructural barrier, rather than the ferrite grain boundary. The postponed repair time will lead to strengthening of the SFC collective effect, so that local microstructure conditions become more and more advantageous for the DSC growth.

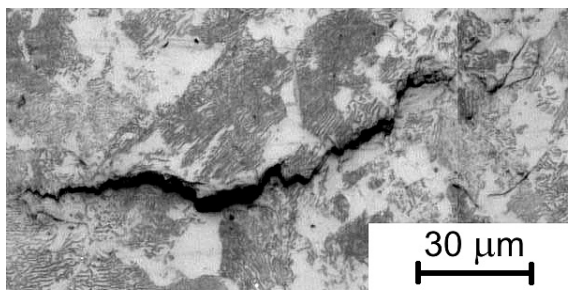

a

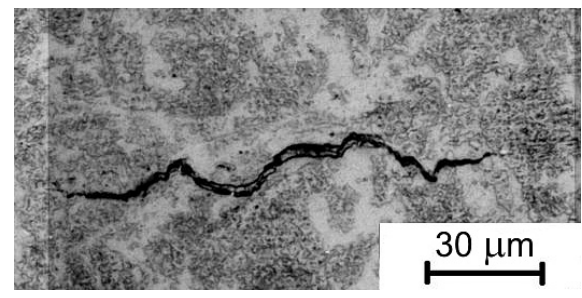

b

Fig. 1. Typical replication photos $(\times 500)$ for specimens in two surface processing states: (a) before maintenance; (b) after maintenance. 
2.3. Fatigue Life. Fatigue life is the most direct indicator of the maintenance effect. To calculate the prolonged-life rate (defined as the ratio of the total number of cycles to the standard life and indicated by $M$ ), the average fatigue life of 137,705 cycles was used as the standard life. Surface turning and rolling treatments significantly extend the fatigue life. The prolonged-life rates for S0.0 to S0.7 specimens are $6.41,5.23,5.06,4.88$, and 4.49, respectively [15].

The maintenance time choice is critical for the life extension effect: the later surface treatment is performed, the weaker the life extension effect may be. This can be attributed to the following factors. Firstly, the fatigue damage accumulated in the material before maintenance cannot be eliminated entirely, so it gets more and more aggravated with delayed maintenance time. Secondly, to remove the existing surface cracks as thoroughly as possible, the thickness of removed layers has to be increased with the postponement of the surface treating time. Thus, the specimen diameter and cross section are also reduced. If short cracks of the same size initiate in two specimens, the local stress in ther specimen with a smaller diameter will be relatively higher.

\section{Revised Short Crack Growth Model.}

3.1. Brief Introduction to Multi-Microstructural Barriers' Model. The multi-microstructural barriers' model was proposed by Yang and Zhao [11]. This model encorporated the effective short fatigue crack criterion and used the characteristic sizes of the material microstructure $d_{1}$ and $d_{2}$. In adition, a resistance coefficient function was introduced to describe the periodic impact of microstructural barriers on the short fatigue crack behavior. The multi-microstructural barriers' model can be defined as

$$
\frac{d a}{d N}=G_{0}+A\left[\Delta W_{t} a-\Delta W_{t} \sum_{i=1}^{n} f_{i}\left(\Delta d_{i}\right) d_{i}\right]^{m},
$$

where $a$ is DSC size, $N$ is the number of cycles, $G_{0}$ is the minimum value of crack growth rate in the first microstructural barrier period, $d_{i}$ is the characteristic microstructural barrier size, $i$ is the subscript to specify the type of barriers, i.e., $d_{1}$ for the average equivalent diameter of ferrite grains and $d_{2}$ for the mean value of intervals between two rich pearlite bands, $A$ and $m$ are the respective material constants, and $f_{i}\left(\Delta d_{i}\right)$ is the resistance coefficient function, which accounts that the closer DSC tip is to the barrier, the stronger is the constraint force. The calculation method for parameters in Eq.(1) has been described elsewhere [11].

3.2. Unified Short Crack Growth Model. If the maintenance process is performed when loading cyclic number is $N_{r}$, the unified short crack growth model for the stages before and after surface treatment can be described as

$$
\frac{d a}{d N}=\langle F(a)\rangle+\left\langle F_{r}(a)\right\rangle
$$

Functions $\langle F(a)\rangle$ and $\left\langle F_{r}(a)\right\rangle$ correspond to short crack growth rates before and after maintenance, respectively. They are defined as

$$
\begin{aligned}
\langle F(a)\rangle & = \begin{cases}F(a), & N \leq N_{r}, \\
0, & N>N_{r},\end{cases} \\
\left\langle F_{r}(a)\right\rangle & = \begin{cases}0, & N \leq N_{r}, \\
F_{r}(a), & N>N_{r} .\end{cases}
\end{aligned}
$$


The residual stress and the hardness of surface vary with the application of maintenance techniques. Furthermore, the accumulated fatigue damage and the microstructural barriers also affect the behavior of initiation and propagation of short fatigue cracks. Hence, the model parameters for specimens before maintenance are no more applicable for description of crack growth after repair, but should be re-calculated based on corresponding test data. Table 1 lists parameters of the dominant short crack growth rate model for typical specimens of LZ50 axle steel at different repair times. Figure 2 illustrates the test data of typical specimens at different maintenance time and the respective predicted curves. It is clear that the present unified short crack growth rate model possesses a good fitting effect to the test data. Moreover, it can reflect the periodic influence of main microstructural barriers on the SFC growth behavior. Comparison between crack growth rate at the same crack driving force show that after maintenance the SFC propagation has sharply decelerated, i.e., the surface turning and rolling tratments effectively suppressed the crack growth.

$\mathrm{T}$ a b 1 e 1

Parameters of Dominant Short Crack Growth Rate Model for Typical Specimens of LZ50 Axle Steel at Different Maintenance Times

\begin{tabular}{|c|c|c|c|c|c|c|}
\hline $\begin{array}{l}\text { Repair } \\
\text { time }\end{array}$ & Stage & $\underset{\mathrm{m}}{d_{1} / d_{r 1}}$ & $\begin{array}{c}G_{01} / G_{0 r 1} \\
\mathrm{~m} / \text { cycle }\end{array}$ & $A_{1} / A_{r 1}$ & $m_{1} / m_{r 1}$ & $\alpha_{1} / \alpha_{r 1}$ \\
\hline \multirow[t]{2}{*}{$f=0$} & Before & - & - & - & - & - \\
\hline & After & $6.13 \cdot 10^{-6}$ & $3.38 \cdot 10^{-11}$ & $2.09 \cdot 10^{-10}$ & 1.15 & 0.69 \\
\hline \multirow[t]{2}{*}{$f=0.3$} & Before & $6.63 \cdot 10^{-6}$ & $2.29 \cdot 10^{-10}$ & $5.75 \cdot 10^{-10}$ & 0.38 & 0.76 \\
\hline & After & $7.76 \cdot 10^{-6}$ & $7.92 \cdot 10^{-12}$ & $9.32 \cdot 10^{-10}$ & 1.25 & 0.62 \\
\hline \multirow[t]{2}{*}{$f=0.5$} & Before & $9.77 \cdot 10^{-6}$ & $3.56 \cdot 10^{-10}$ & $4.73 \cdot 10^{-9}$ & 0.51 & 0.66 \\
\hline & After & $7.78 \cdot 10^{-6}$ & $9.96 \cdot 10^{-12}$ & $5.52 \cdot 10^{-10}$ & 1.47 & 0.52 \\
\hline \multirow[t]{2}{*}{$f=0.6$} & Before & $6.6 \cdot 10^{-6}$ & $2.94 \cdot 10^{-10}$ & $5.46 \cdot 10^{-9}$ & 0.48 & 0.79 \\
\hline & After & $6.78 \cdot 10^{-6}$ & $4.19 \cdot 10^{-11}$ & $6.33 \cdot 10^{-10}$ & 1.54 & 0.64 \\
\hline \multirow[t]{2}{*}{$f=0.7$} & Before & $8.4 \cdot 10^{-6}$ & $5.17 \cdot 10^{-10}$ & $1.74 \cdot 10^{-9}$ & 1.46 & 0.67 \\
\hline & \multirow[t]{2}{*}{ After } & $8.27 \cdot 10^{-6}$ & $1.21 \cdot 10^{-10}$ & $5.33 \cdot 10^{-10}$ & 1.30 & 0.62 \\
\hline & & $\begin{array}{c}d_{2} / d_{r 2} \\
\mathrm{~m}\end{array}$ & $\begin{array}{c}G_{02} / G_{0 r 2} \\
\mathrm{~m} / \text { cycle }\end{array}$ & $A_{2} / A_{r 2}$ & $m_{2} / m_{r 2}$ & $\alpha_{2} / \alpha_{r 2}$ \\
\hline \multirow{2}{*}{$f=0$} & Before & - & - & - & - & - \\
\hline & After & $6.69 \cdot 10^{-5}$ & $2.59 \cdot 10^{-10}$ & $6.28 \cdot 10^{-11}$ & 1.12 & 0.57 \\
\hline \multirow[t]{2}{*}{$f=0.3$} & Before & - & - & - & - & - \\
\hline & After & $5.85 \cdot 10^{-5}$ & $6.96 \cdot 10^{-10}$ & $8.37 \cdot 10^{-11}$ & 1.43 & 0.50 \\
\hline \multirow{2}{*}{$f=0.5$} & Before & - & - & - & - & - \\
\hline & After & $6.05 \cdot 10^{-5}$ & $1.46 \cdot 10^{-9}$ & $5.84 \cdot 10^{-11}$ & 1.34 & 0.53 \\
\hline \multirow[t]{2}{*}{$f=0.6$} & Before & - & - & - & - & - \\
\hline & After & $6.0 \cdot 10^{-5}$ & $8.95 \cdot 10^{-11}$ & $3.57 \cdot 10^{-11}$ & 1.32 & 0.42 \\
\hline \multirow[t]{2}{*}{$f=0.7$} & Before & $5.57 \cdot 10^{-5}$ & $4.82 \cdot 10^{-10}$ & $3.45 \cdot 10^{-10}$ & 1.22 & 0.56 \\
\hline & After & $4.49 \cdot 10^{-5}$ & $8.65 \cdot 10^{-10}$ & $1.99 \cdot 10^{-11}$ & 1.38 & 0.45 \\
\hline
\end{tabular}



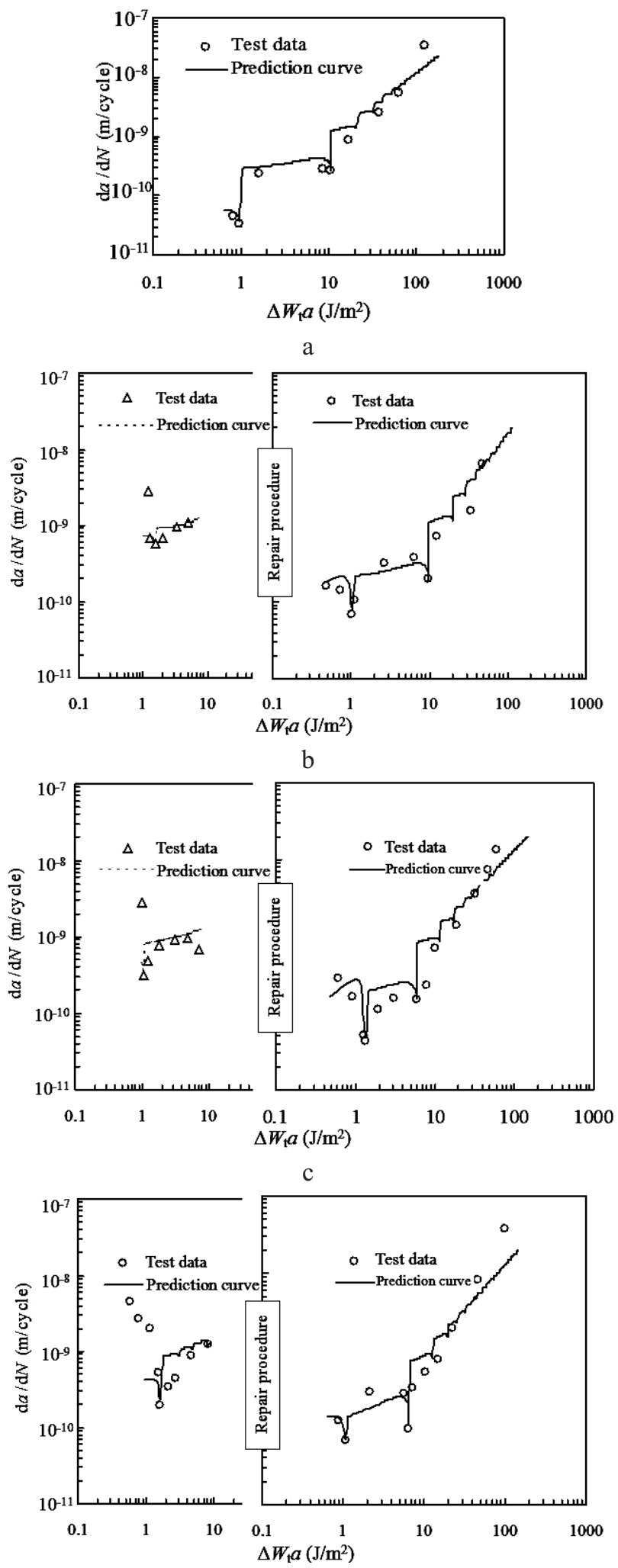

d 


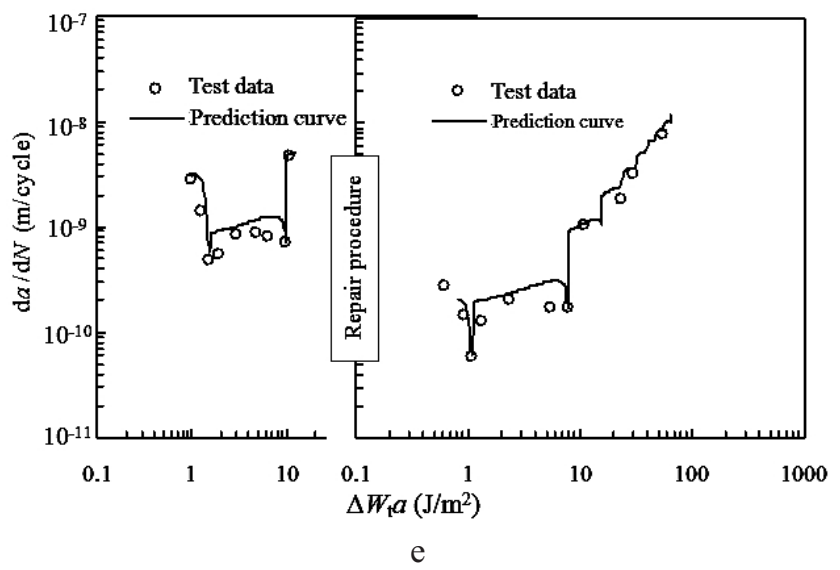

Fig. 2. Dominant short crack growth rate curves for typical specimens of LZ50 axle steel at different maintenance times: (a) $f=0$; (b) $f=0.3$; (c) $f=0.5$; (d) $f=0.6$; (e) $f=0.7$.

3.3. Modified Multi-Microstructural Barriers' Model. According to Section 2.3, the prolonged-life rate $M$ decreases from 6.41 to 4.49 due to the postponed maintenance time. It is easy to conclude that in the extreme case of $f=1$, specimens would be damaged without any surface treatment and the prolonged-life rate would be equal to 0 . Therefore, the maintenance time effect function can be constructed as

$$
M(f)=K+D(1-f)^{C},
$$

where $K, D$, and $C$ are the material constants. As mentioned above, in case of $f=1$ and $M=0$, Eq. (5) can be reduced to

$$
M(f)=D(1-f)^{C} .
$$

Values of $D$ and $C$ for the present data are 6.411 and 0.475 , respectively.

To extend the applicable scope of the previous model and utilize the test data effectively, the maintenance time effect function can be introduced to modify the multimicrostructural barriers' model. With the given DSC size, the growth rate of a specimen without maintenance is higher than that of a rolled specimen. After the same number of loading cycles, DSC increment of the latter specimen is larger. Transformation of the prolonged-life rate into the crack growth rate variation is depicted in Fig. 3, which shows the process of data modification. It is seen that, after shifting the test data of specimens without maintenance by the factor of 6.41, the growth rates for unrolled specimens and S0.0 specimens overlapped. Similar results can be obtained by reducing the growth rate of unrolled specimens by the value of $M$, according to the maintenance time (Fig. 4). Therefore, a modified multi-microstructural barriers' model can be expressed as

$$
\frac{d a}{d N}=\frac{1}{M(f)}\left\{G_{0}+A\left[\Delta W_{t} a-\Delta W_{t} \sum_{i=1}^{n} f_{i}\left(\Delta d_{i}\right) d_{i}\right]^{m}\right\} .
$$

Thus, after the test data on specimens without maintenance is obtained, the equation controlling the crack growth rate for specimens with any maintenance time can be derived from Eq. (7). This modified model can be quite convenient for assessment of the remaining fatigue life and evaluation of the structural safety. However, the repair process enhances 


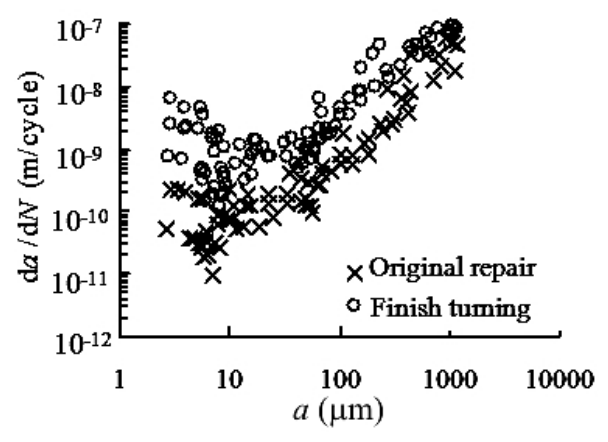

a

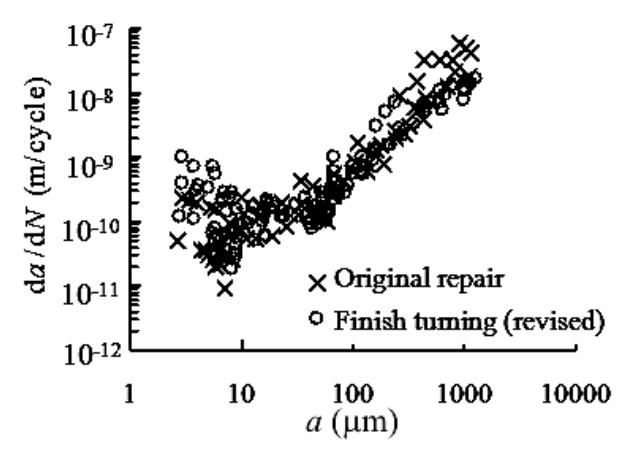

$\mathrm{b}$

Fig. 3. Data comparison between finish-turned specimens before/after data the initial (a) and modified (b) repaired specimens of LZ50 axle steel.

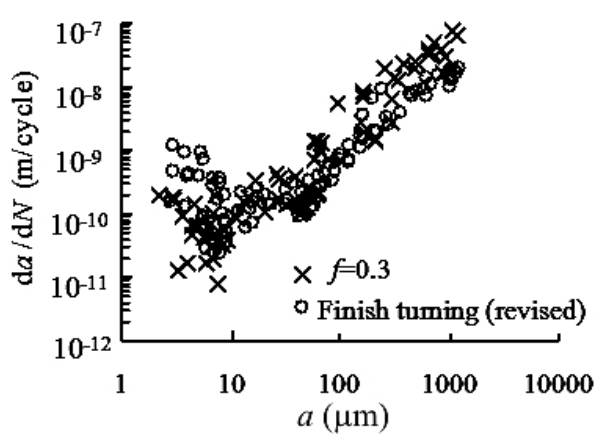

a

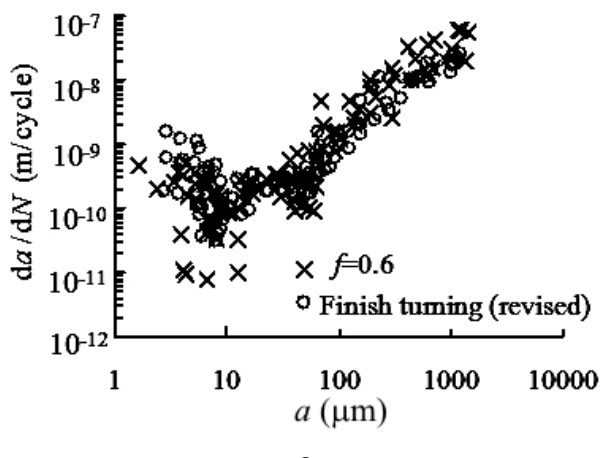

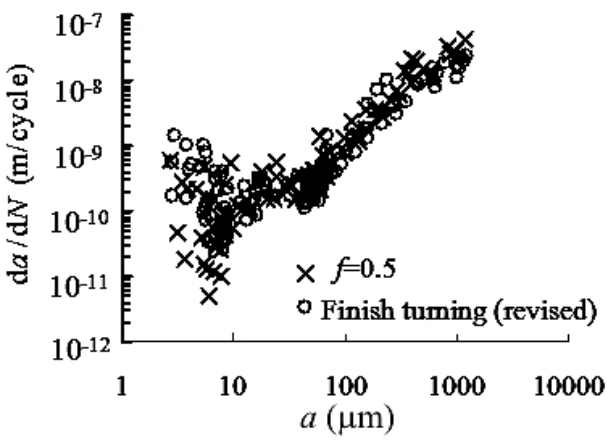

$\mathrm{b}$

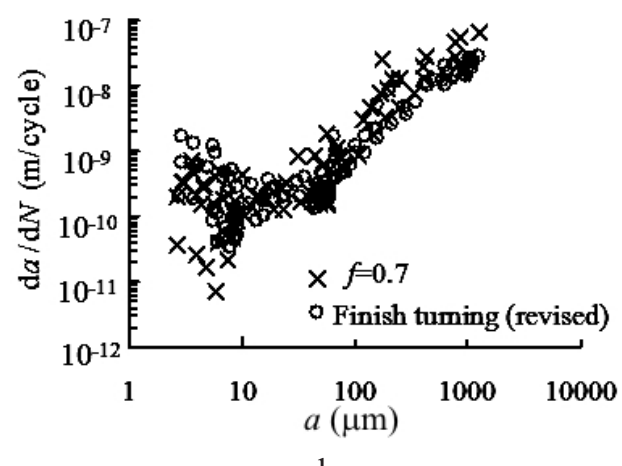

d

Fig. 4. Comparison between modified data on finish-turned specimens and data on specimens of LZ50 axle steel at different maintenance times: (a) $f=0.3$; (b) $f=0.5$; (c) $f=0.6$; (d) $f=0.7$.

crack propagation at the PSC stage, as compared to the unrolled specimen, which issue requires a further investigation.

4. Discussion. The two primary microstructural barriers to short fatigue crack growth in LZ50 axle steel are ferrite grain boundary and rich pearlite banded structure. The DSC growth rate twice manifest a drop at the MSC stage due to the above microstructural barriers. The crack growth rates in specimens after surface turning and rolling are significantly lower than those in specimens before maintenance.

The effective short crack density values exhibit an initial rise, followed by a drop, for all five groups of specimens. After maintenance, the density values are significantly 
reduced. Surface rolling effectively restrains the initiation of collective short cracks. However, this effect is weakened if the maintenance time is postponed.

The maintenance process significantly prolongs the fatigue life, because the turning treatment removes the existing short cracks from the material surface, while rolling improves the fatigue performance of the new surface. However, postponed implementation of maintenance inhibits elimination of the accumulated fatigue damage. Moreover, the required increase in the cutting thickness also increases the actual local stresses.

Conclusions. A short crack growth model is proposed, which is based on modification of the multi-microstructural barriers' model and adequately describes the deceleration behavior of growth rate during the whole SFC propagation process. By introducing a maintenance time effect function, the model can predict the growth rate in specimens with any maintenance time based on the available test data on specimens without maintenance. The proposed model can be instrumental for the assessment of remaining fatigue life and the structural safety evaluation.

Acknowledgments. This study is supported by the National Natural Science Foundation of China (51205326 and 51275432), the Fundamental Research Funds for the Central Universities (SWJTU11CX075), and the Opening Project of State Key Laboratory of Traction Power (Grant No. 2011TPL_T02).

1. Z. Y. Shen, Y. X. Zhao, B. Yang, and J. C. Peng, "Progresses on the fatigue reliability research of China railway," Adv. Mater. Res., 44-46, 1-14 (2008).

2. Y. X. Zhao, Q. Gao, B. Zhang, and K. J. Diao, "Key solid mechanics issues and research progresses for railway vehicle wheelset," Chin. J. Solid Mech., 31, No. 6, 716-730 (2010).

3. S. Pearson, "Initiation of fatigue cracks in commercial aluminum alloys and the subsequent propagation of very short cracks," Eng. Fract. Mech., 7, 235-247 (1975).

4. Y. X. Zhao, B. Yang, and M. F. Feng, "Critical safety fatigue crack sizes for the RD2 type axle of Chinese railway freight car," in: S. J. Wu, P. E. J. Flewitt, Z. Zhang (Eds.), Proc. 9th Int. Conf. on Engineering Structural Integrity Assessment (Beijing, China, 2007), China Machine Press (2007), pp. 1194-1199.

5. Y. X. Zhao, B. Yang, M. F. Feng, et al., "Probabilistic critical fatigue safety state of the RD2 type axle of China railway freight car," Adv. Mater. Res., 44-46, 751-758 (2008).

6. M. W. Brown, "Interfaces between short, long and non-propagating cracks," in: K. J. Miller and E. R. de los Rios (Eds.), The Behavior of Short Fatigue Cracks, Mechanical Engineering Publications, London (1986), pp. 423-439.

7. P. D. Hobson, M. W. Brown, and E. R. de los Rios, "Two phases of short crack growth in a medium carbon steel," in: K. J. Miller and E. R. de los Rios (Eds.), The Behavior of Short Fatigue Cracks, Mechanical Engineering Publications, London (1986), pp. 441-459.

8. K. J. Miller, H. J. Mohamed, and E. R. de los Rios, "Barriers to short fatigue crack propagation at low stress amplitude in a banded ferrite-pearlite structure," in: R. O. Ritchie and J. A. Lankford (Eds.), Small Fatigue Cracks, Lankford Publication of the Metallurgical Society, Inc., PA (1986), pp. 639-656.

9. A. Navarro and E. R. de los Rios, "A model for short fatigue crack propagation with an interpretation of the short-long crack transition," Fatigue Fract. Eng. Mater. Struct., 10, 169-186 (1987).

10. J. Polak and P. Liskutin, "Nucleation and short crack growth in fatigued polycrystalline copper," Fatigue Fract. Eng. Mater. Struct., 13, 119-133 (1990). 
11. B. Yang and Y. X. Zhao, "Experimental research on dominant effective short fatigue crack behavior for railway LZ50 axle steel," Int. J. Fatigue, 35, 71-78 (2012).

12. Z. He and W. W. Yao, "Rotating bending fatigue property of LZ50 steel axle," Mater. Mech. Eng., 36, 94-96 (2012).

13. B. Yang and Y. X. Zhao, "Influences of final processing methods on surface physical properties and fatigue life for railway LZ50 steel," Adv. Mater. Res., 463-464, 85-89 (2012).

14. M. H. Swain, "Monitoring small-crack growth by the replication method," in: J. M. Larsen and J. E. Allison (Eds.), Small-Crack Test Methods, ASTM STP 1149, Philadelphia (1992), pp. 34-56.

15. B. Yang and Y. X. Zhao, "Influence of surface rolling time on short fatigue crack behavior of LZ50 axle steel," in: Proc. 13th Int. Conf. on Fracture (Beijing, China, 2013), pp. 1774-1781.

16. Y. X. Zhao, Q. Gao, and J. N. Wang, "Interaction and evolution of short fatigue cracks," Fatigue Fract. Eng. Mater. Struct., 22, 459-468 (1999).

17. Y. X. Zhao, "Size evolution of the surface short fatigue cracks of $1 \mathrm{Cr} 18 \mathrm{Ni}$ Ti pipe-weld metal," J. Mater. Sci. Technol., 19, 129-132 (2003).

18. B. Yang and Y. X. Zhao, "Surface rolling effect on effective short fatigue cracks density for railway LZ50 axle steel," Adv. Mater. Res., 118-120, 75-79 (2010). 